\title{
Particle-Enhanced Turbidimetric Immunoassay
}

National Cancer Institute

\section{Source}

National Cancer Institute. Particle-Enhanced Turbidimetric Immunoassay. NCI

Thesaurus. Code C125953.

A method to quantify the opaqueness of a liquid using a turbidimeter, by measuring the decrease in light intensity due to the scattering effect of suspended immunoaggregates in a liquid sample. The immunoaggregates are target antigens agglutinated to antigenspecific antibody-coated polystyrene latex-enhanced particles. 